\title{
Evolution Characteristics and the Mainstream Pattern Analysis of MOOCs in Chinese Higher Education
}

\author{
Jing DU1', Fei LI¹, Bin LI¹ ,Lei YANG² \\ 1. Information Technology Center \\ Tsinghua University \\ Beijing, China \\ 2. Academic Affairs Office \\ Tsinghua University \\ Beijing, China \\ E-mail: dujing@tsinghua.edu.cn; ylei@tsinghua.edu.cn
}

Keywords: Online Courses; MOOCs; OER; Pattern Analysis

\begin{abstract}
First of all, paper is proposed that there are 19 components and 6 evolution characteristics of the online courses, and that those components are classified into course overview information, self learning elements, learning feedback elements, and cooperation\& interaction respectively. Then, the three mainstream patterns of online courses in China are analyzed in the Massive Open education context. By comparing and analyzing the characteristic components, learning content organization and course orientation, the 4 construction principle of online courses are proposed, including the modular organization and course development. This paper aims to not only provide the course designers and developers with ideas, but also improve the efficiency of online course design and construction.
\end{abstract}

\section{Components and Evolution Characteristics of Online Courses}

The online courses in the Massive Open education context are publicly accessible through the Internet. They can provide interaction participated by a huge number of learners.

The 19 components of online courses may be classified into four categories from the perspective of learners, as shown in the TABLE I. 


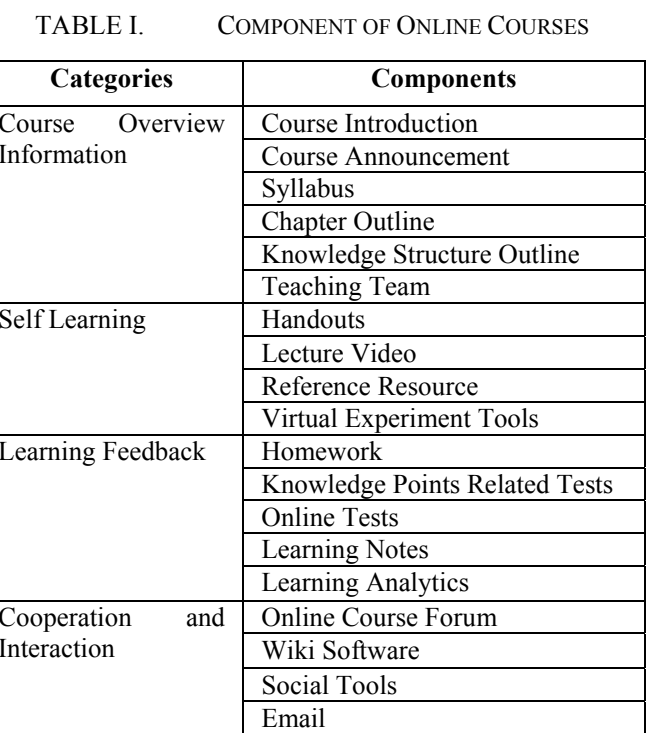

\section{Evolution Characteristics of Online Courses}

In addition to such course resources as lecture video, reference material and online tests, the online courses in the Massive Open education context also provide interactive user forums, establish web-based learning community for learners, teachers and teaching assistants, and adopt the big data technology to process the learning data, thus providing information feedback for both teachers and learners. Driven by the information technology, data analysis technology and information literacy promotion of net citizens, the following evolution characteristics are developed in the online courses.

\section{Huge participant at diverse levels}

In this Internet era, people are becoming as familiar with online education as online shopping and online games. The wide-open online courses allow everyone to access to the learning opportunities easily. There is no threshold in the wide-open online courses, which leads to a huge number of participants at diverse levels of ages, cognitions and learning demands.

\section{Well-made content and be wide-open}

The online courses in the Massive Open education context share the characteristics with mass media. With special or well-received contents taught and the course structure re-designed, the teaching contents are classified more rationally, and the courses are more suitable to online learning. The well-made digital courses are produced by a professional team and published on the open platform accessible by the public. The online courses are different from the traditional ones in the well-made content and wide-open access.

\section{Modular organized micro-video and fragmented contents}

The online learning process is random and fragmented, so the existing online courses are usually micro videos, not only following learners' browsing habits and concentration rules, but also satisfying the learners' demands for dispersed learning time, thus realizing the personalized learning process.

In order to prevent the learners from getting lost in the vast amounts of micro videos, the contents of online courses are modular organized, and the sequence of learning resources is designed by certain logical order, thus making the sequence of learning tasks multiple-entry but orderly. 


\section{Online learning activities with social interaction}

In the online courses in the Massive Open education context, the course forums are used to realize the remote interaction and collaboration between teachers and learners. The online learning activities are active on a regular basis, because the participants tend to have a large-scale participation in a fixed time. Wiki Software, learning notes and other knowledge construction tools are involved to guide the participants to contribute valuable learning resources and learning experiences at any time, as amended and discussed in groups from time to time, thus forming the knowledge base for online courses.

\section{Online automatic evaluation and peer review}

The large amount of learners leads to the dramatically increase in student/teacher ratio, so the online courses may provide automatically evaluated in-class tests and examinations. With the questions and answers preset in the system, after acquiring the interpretation of a knowledge point, the students may participate in the online test and compare their answers with the references preset in the system, thus promptly checking their mastery of that knowledge point. The database may be used for midterm examination and final examination. The learners may take the test in the good faith, and the system will evaluate their results automatically and give a score. The test score given by the database may be an important examination level for the learning quality of learners.

Peer review is applicable to the MOOCs, which is facilitated by the large amount of participants and their sense of belonging to the Internet. In the process of peer review, the learners may not only know the learning of other peers, but also improves the personal knowledge reflection and promotes the formation of critical thinking. In addition to enriching personal cognition, the learners participating in peer review may become fonder of learning with less learning anxiety alone.

\section{Learning Analytics by big data technology}

The online learning environment is provided in the MOOCs. All online learning activities of learners will be recorded or collected by the learning platform. A large amount of learning data has been accumulated in the MOOCs due to the numerous participated, which has provided the foundation of online learning analytics.

The online learning analytics shall be conducted from various perspectives for the online learning data under the guidance of certain education goals, and then the analytics results will be promptly fed back to the learning process, thus planning, reviewing, evaluating, feeding back, controlling and regulating the whole online learning process. Visualization technology may be used in the learning analytics to display the abstract results vividly for the better understanding of users, thus better regulating and controlling the online education process.

\section{Analysis of Mainstream Pattern of Online Courses in China}

Based on the components of online courses above, the three mainstream patterns of online courses in China are analyzed, although they are different in components and characteristics to some extent. The three mainstream patterns are represented by Chinese University open courses, resource sharing courses and personal social learning online courses.

\section{Patten I: Lecture-based Online Courses - Chinese University Open Courses Project}

Lecture-based online courses usually explain the classic or well-received contents in the form of easyto-read lectures, of which the videos produced by the publishing levels are released on the Internet. Mainly represented by China University Open Courses (shown in Figure 1) and Netease Public Class Project, these courses not only reflect the social responsibility of famous teachers and courses to serve the public, but also promote the cultural communication and the popularization of scientific knowledge. 
The core components of these online courses are course information, lecture video, chapter outline, comments, and teaching team.

- Course information: range of course, characteristics and cultural or subject features.

- Courseware introduction: content introduction and culture interpretation of each courseware.

- Lecture video: core contents of each lecture, direct on-line learning without registration, usually lasting for 30 to 45 minutes.

- Comments: comments made by any video viewers after registration.

- Learning record: recording the videos watched already and the watching time respectively.

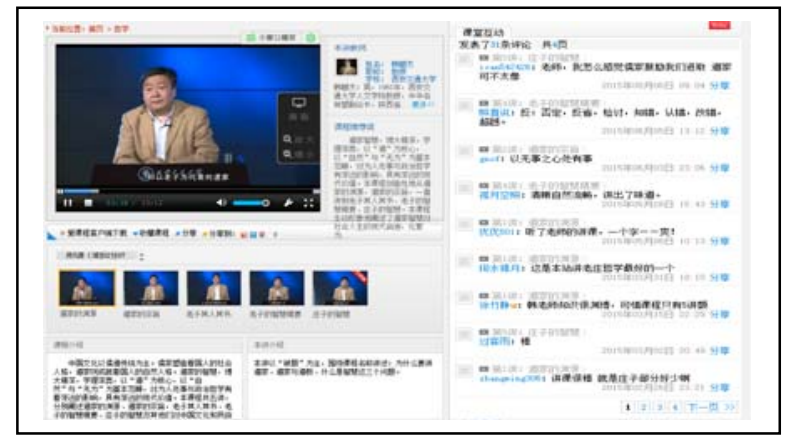

Figure 1. Interface of Chinese University Open Courses

\section{Patten II: Teacher-led Online Courses - Resource Sharing Courses Project}

With the traditional face-to-face classroom teaching process as the main line and the course contents classified by chapter structure, the teacher-lead online courses make the learning resources in each faceto-face teaching link digitized and put on the online learning platform. Represented by the Resource Sharing Courses Project in the universities in China(shown in Figure 2), such courses emphasize that the learning process shall be lead by top teachers with the help of teaching team, so as to construct an online learning community which supports the learners to interact through formal learning, thus constructing the knowledge.

The core components of these online courses are:

- Course information: course name, teachers, subject category, applicable majors, course introduction, teaching outline, course agenda, evaluation, study guides;

- Chapter outline: the major classification manner of course contents in the teacher-lead online courses, in which the course contents are classified by teachers in the face-to-face class as per the chapters;

- Knowledge structure outline: the summary of knowledge points formed by teachers through annotating the videos, assignments, and handouts, which is convenient for users to quickly find all teaching resources associated with a certain knowledge point.

- Teaching team: introduction of team members and their resumes;

- Handouts: teacher's handouts in the face-to-face class synchronously quoted into the online courses, which are organized as per the chapter outline to;

- Lecture video: the teacher's teaching video in the face-to-face class, which is supplemented by handout picture and scene display picture to highlight the leading by top teachers, and published on the online learning platform as per the original chapter order with teacher's course design as the main line, usually lasting for 45 minutes;

- Reference books: paper reference books and relevant materials recommended by teachers in the course learning process, such as thesis, book checklist, etc.; 
- Course forum: supporting the online comments, the posting of self learning experience, and the asynchronous discussion of learning contents;

- Case library: added in some courses to enrich the course contents for users;

- Homework: although assigned as per the chapter structure, only the title and corresponding files indicated in the teacher-led online courses, making the online tests and automatic feedback not available;

- Learning notes: allowing the users to make learning notes online when studying the lecture video, and to share such learning notes with others studying the same teacher-led online course.

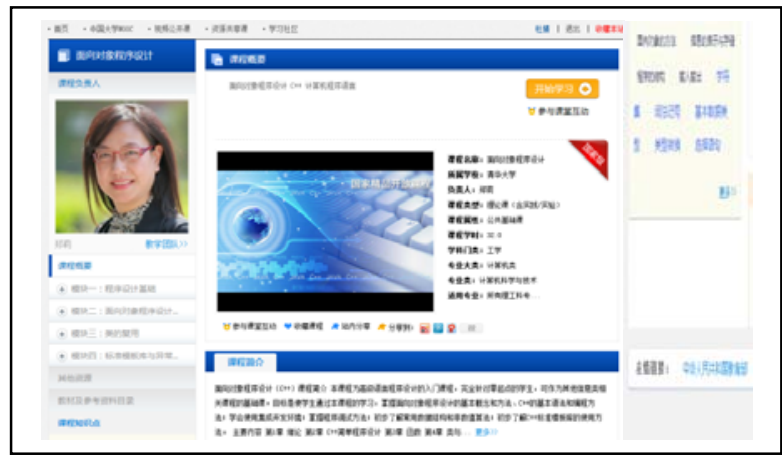

Figure 2. Interface of Resource Sharing Courses

\section{Patten III : Personal social learning Online Courses}

Personal social learning Online Courses consist of instructional design prepared by teaching team using excellent course content, more exact and organized content by course producers with the advanced method, and online automatic evaluation and test, online virtual experiment. These courses support persons' learning by themselves, and support persons' working together.

This kind of online courses is not given by only one teacher, but by several. Breaking the teaching mode of face-to-face courses, these online courses enable learners and teachers to construct an online learning community, where learners can learn courses by themselves and interact with others. The learning platform affords network socialization, thus making it possible for learners to work with partners, and achieving the effect of the socialized construction of knowledge. The online learning system working in this mode provides feedback learning record to ensure the accomplishment ratio of the courses(shown in Figure 3).

This kind of online courses focuses on learners' autonomy and social networking learning. The curriculum elements of them are as follows:

- Course bulletin: teaching team gives notice of course content progress and demand, and delivers dynamic announcement of courses with the processing of course-teaching.

- Course information: syllabus, course introduction, teaching calendar, handouts and the related website address

- Teaching week and knowledge point sequence: teaching content of MOOCs is divided into several parts on a teaching week basis. Teaching team determines the teaching content of one teaching week based on its chapter structure. The teaching content of one week includes learning material of several knowledge points, such as lecture micro-video and its corresponding quiz

- E-textbook: e-textbooks and e-books.

- Wiki software: knowledge sharing and managing space. It stores material teaching team think important, the word documents accomplished by several learners during the process of online cooperation, and outstanding academic achievements by learners. Wiki software support learning 
and word document editing by several learners in a cooperative way. Therefore, learners can participate in a particular learning group according to their interest and learn with several learners.

- Course forum: in the course forum, knowledge point, a basic element of course, can be talked about. Content of teaching resources can also be talked about. The forum supports cooperative learning, which facilitates knowledge construction and cooperative learning.

- Lecture video: course overview video and course content video. In the former video, the teacher team introduces to the learners course information and assessment requirements through the lens, while the latter is a micro video of 15 minutes, each video explaining one knowledge point. Course content video also contains advice on learning.

- Virtual experiment tools: for science and engineering courses, it will provide online virtual experiment environment. To be specific, it will provide opportunities for learners to conduct online experiments, which can deepen the understanding of knowledge.

- Online Quiz and Tests: Teachers can set knowledge related online quiz and tests in the lecture video sequences. MOOCs Learning platform provides automatic feedback when learners finish their test.

- Learning analytics: Statistics for individual learners' schedule in each course, including the number of videos of each teaching week, homework score record, and test accuracy record. Learners even can see their ranking place of all students learning the same courses. Record and analysis of data in learning process can enable them to adjust their learning plan, thus making learning more effective.

- Learning notes: learners can take online notes when listening, and also can share notes with other learners.

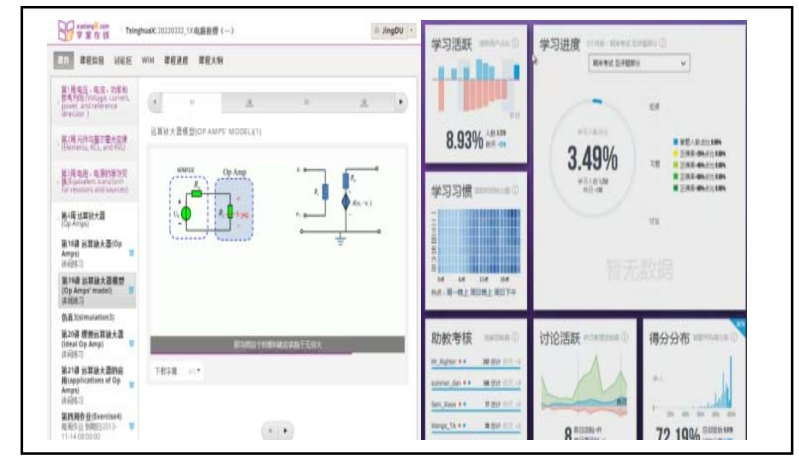

Figure 3. MOOCs Interface and Learning Analytics Result

\section{Comparison of the Three Online Course Patten}

course orientation

- Patten I: Free for anyone even no registration

Lecture-based online courses are provided by government or public organizations. They are free for anyone even with no registration.

These courses are aimed at the public wanting to know scientific and cultural knowledge in certain field, with the purpose of promoting the cultural development and spiritual progress.

- Patten II: Free for registered students and subject teachers

These courses are aimed at face-to-face course required learners. With the power of rebuilding the situation of giving face-to-face courses, they enable online learners to grasp the knowledge obtained from 
face-to-face courses. These courses are also provided for teachers expecting to improve teaching ability. Such courses offer the opportunity for these teachers to appreciate the teaching process of famous university teachers, and learn methods and skills in instructional design of this subject, thus promoting the teaching ability of teaching teams. Teacher-led online courses can facilitate the sharing and exchange of teaching resource between different schools.

- Patten III: Free for registered customers but pay for certification

This category of online courses is generally provided by commercial online education institutions. They are free for registered customers but certification is not. These courses are aimed at those having higher information literacy, having good network condition, familiar with the way of social networking, and not convenient for participating face-to-face courses. These courses make it convenient for them to make use of fragmented time to learn knowledge. For this kind of courses, certification to be signed and approved can be offered by the teachers of the course. The certificate is charged of a small fee. At the beginning of the course, learners can choose whether to apply for a certificate, as certificate candidates need to go through strict process assessment. Not only online homework and online quiz are required, but also the final assessment of the course is needed (which may be the online exam, or maybe off-line one), to obtain a certificate.

\section{characteristic components}

The three kinds of courses are different in characteristic components. To be specific, Patten I contain 7 elements to ensure that the audience can easily access to the course content, but less learning feedback channels are provided. Patten II includes 11 elements, which can support learners' online learning courses, and provide limited collaborative communication and learning feedback. Patten III contains all the 19 elements of the online courses, which can provide the service of learning, collaboration, knowledge construction, and learning feedback and analysis from the four aspects of autonomous learning, learning feedback, collaborative communication and course overview.

The core components of these three kinds of courses are compared as Figure 4:

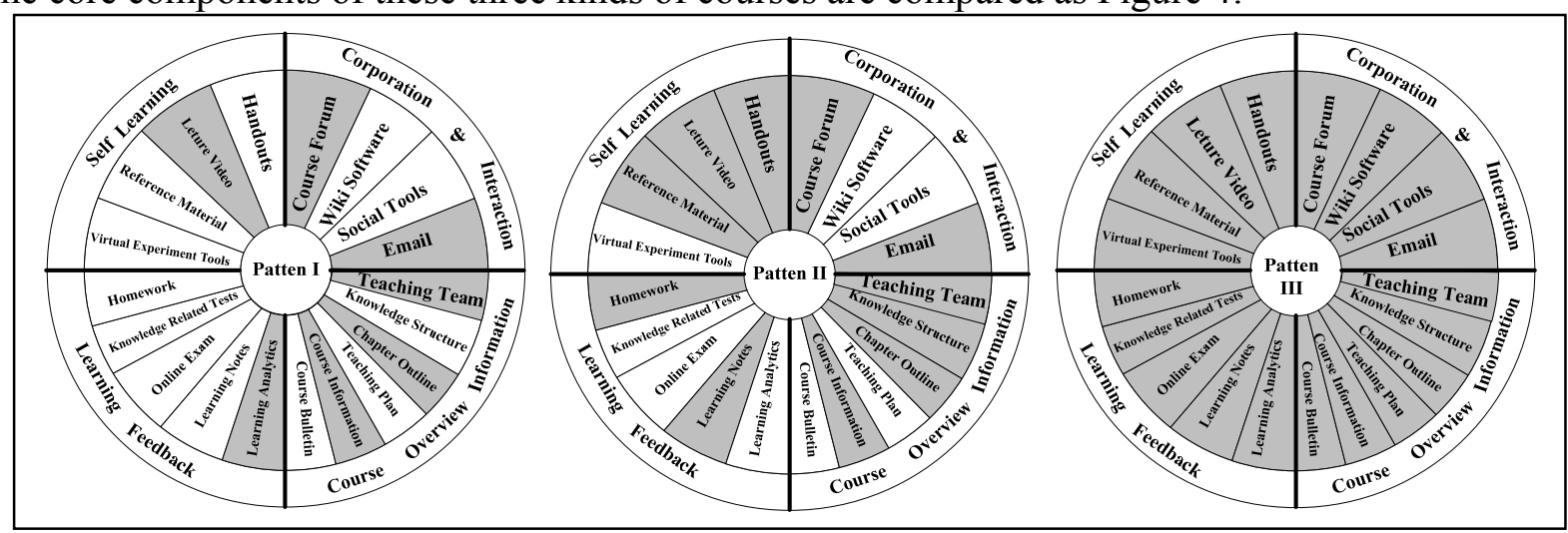

Figure 4. Core Component Comparison of Three Pattens

\section{Learning content organization}

- Patten I: Each video has duration of 30 minutes. No chapter outline, no knowledge Structure

The lecture content is organized based on one topic. Each topic contains many independent videos, each about 30 minutes, which can meet the requirement of sharing and openness of open courses. To restore the infection and affection of lecture, broadcasting producing mode is applied. These courses play a pivotal role in famous universities promoting social culture and spreading scientific knowledge. 
- Patten II: Each video has duration of 45 minutes, to rebuild face-to-face classes, with chapter outline, and primary knowledge structure

Teacher-led online courses highlight that teachers guide the learning process through the curriculum design. Most of the teaching resources are divided according to the chapter structure of paper textbooks. Course video generally has duration of 45 minutes, and stored in the same file with the corresponding handouts, assignments, quiz documents and other illustrating documents. But at the end of each chapter, the main points corresponding to the content of the leaf node are indicated. All of knowledge points form an unstructured knowledge point set, displayed in the course homepage, which makes it convenient for users to search for the corresponding teaching content.

For teacher-led online courses, a primary double-retrieval method for organizing teaching content is used. And the two retrieval methods are chapter structure and knowledge point set, respectively.

- Patten III: Each video has duration of less than 15 minutes. Double-retrieval method (chapter of each teaching week, and knowledge point). Support online collaborative learning.

For this kind of online courses, a double-retrieval method (a combination of chapter of each teaching week, and knowledge point) is used to organize the teaching content. For each course, the teaching content in each teaching week is determined, and it contains a lot of teaching resource related to knowledge points within this week, including lecture video, in-class test, handouts, forum topic, reference resource, and virtual experiment.

The teaching content of each teaching week is independent on one hand, but connected with that of others on the other hand, ensuring the integrity and systematicness of the course. Modular organization can be helpful for students in establishing the connection of different knowledge and mastering course knowledge from the perspective of the whole system.

By knowledge points in each teaching week sequence method and by using strong logical teaching week module, fragmented learning resources can be put together. This kind of courses not only supports cognitive habit of network fragmentation, but also provides system structure through the learning platform. Lecture video has duration of less than 15 minutes, which makes it easy for learners to make use of scattered time to study, and not go astray in the course of learning.

\section{Conclusion: Construction Principle of Online Courses}

\section{Content organization}

The integral course structure should be clear in logics. It can be divided into a four-layer structure by gradual refinement. The four-layer structure can be course (lecture), chapter (module), section (special topic) and small section (knowledge point) from top to bottom. Course is the first-step structure. It is a structure of course layer divided by subject content. The first layer includes element like course information; chapter is second-step structure. It is a structure of modularization formed by the classification of teaching task, which includes elements like course information and main contents of course. Section is the third-step structure, which makes special classification toward teaching contents in units of chapter and forms gatherings of knowledge points. It includes a series of learning activities. Small section is the smallest structure, which is teaching content divided according to knowledge points under the layer of section. It includes all kinds of learning resources such as course video, quiz, lecture notes, electronic teaching materials, exercises, and experiment guidance.

The teacher can choose the above four-layer structure or a structure smaller than it according to his own decision to design course based on features of subject contents.

As for the same course, all kinds of teaching resources and materials can be divided by following the same-layer structure. Contents under the same-layer structure can be a system of its own, or they can 
mutually be related under the same-layer structure. In order that students can learn online-course, the teacher should classify course contents toward the smallest-layer structure by using knowledge points or skill points as far as possible.

The four layers and content partition of course structure design can be shown in the TABLE II:

\begin{tabular}{|c|c|c|c|c|}
\hline Graininess & Layer name & Another name & Introduction of layer graininess & Partition of teaching contents. \\
\hline $\begin{array}{l}\text { The top } \\
\text { layer }\end{array}$ & course & lecture & $\begin{array}{l}\text { A structure of course layer divided according } \\
\text { to subject contents }\end{array}$ & Including element like course information \\
\hline Large & Chapter & Module & $\begin{array}{l}\text { a structure of modularization formed by the } \\
\text { classification of teaching task }\end{array}$ & $\begin{array}{l}\text { including elements like course } \\
\text { information and main contents of course }\end{array}$ \\
\hline Medium & Section & Special topic & $\begin{array}{l}\text { It makes special classification toward teaching } \\
\text { contents in units of chapter and forms } \\
\text { gatherings of knowledge points. }\end{array}$ & Including a series of teaching steps \\
\hline small & small section & $\begin{array}{l}\text { Knowledge } \\
\text { points }\end{array}$ & $\begin{array}{l}\text { teaching contents divided according to } \\
\text { knowledge points }\end{array}$ & All kinds of resources \\
\hline
\end{tabular}

Based upon the above structure design and content partition, all teaching activities of online course are composed by different learning resources. Toward course structure of each layer, the teacher should properly add interaction steps. For example, when he makes knowledge explanation in each teaching activity, he can match to use online test and discussion to help learners timely feedback contents.

\section{Principles of course research and development}

Toward elements and features of the above online courses, we should follow the following principles in developing online courses.

1) We should make sure that contents can swiftly be updated.

MOOCs have a high application rate, a large page view and a short course period. These require the course developers to form an online course within a short period of time and based on existing digitalized teaching resources. Course contents should also swiftly be updated and existing courses should be on line as soon as possible.

2) We should lay emphasis on learning activity design

Under the learner-oriented teaching ideology and for the sake of students' higher learning efficiency on line, the teacher should mainly embody his teaching ideas in designing learning activity. Learning activity design is the core part of online course design and it is expressed on offering students learning task order. Thus, it enables students to keep learning concentration on line and to improve their learning efficiency.

3) We should support innovation of teaching design

Construction of online course is not a thing for good and all. Course and teaching design should constantly and dynamically recombined and generated with teaching reform. Therefore, when we do construction of online courses, we should consider supporting innovation of teaching design and dynamic generation of online courses in aspects like planning forms.

4) We should offer process data to make analysis

An important step of teaching process is teaching feedback. Teaching feedback of online courses originates from data analysis toward the online learning process. Therefore, we should offer related teaching activity data to make feedback and learning analysis. 


\section{References}

[1] Y. Wang, J. Zhang, B. Zhang," MOOC: Characteristics Analysis Based on Typical Projects and Its Enlightenment”, Journal Of Distance Education, vol.4, pp.67-65, April 2013.

[2] L. Yuan, S. Powell, H. Ma, “Analysis of Massive Open Online Courses Initiatives", Open Education Research, Vol.19, No. 3, pp56-62, June 2013.

[3] Q. Li, Z. Hou, T. Wang, "Bussiness Model of MOOCs", Open Education Research, Vol.19, No. 5, pp71-78, Oct. 2013

[4] P. Wang, "The Latest Development and Application of Massive Open Online Course: From cMOOC to xMOOC", Modern Distance Education Research, Vol. 123, pp13-19, March 2013.

[5] X. Zhang, D. Li, L. Chen, “The Attention Guidance Design of Micro-lecture Video Processing”, Modern Educational Technology, Vol. 23, pp105-107, August 2013

[6] Larry,C, ".MOOCs and pedagogy:Teacher-centered,student-centered,and hybrids (Part 1)", http://larrycuban.wordpress.com/2013/02/13/MOOCs-and-pedagogy-part-2/ . 2012

[7] Siemens G,"How to Participate in an Open Online Course", http://gsiemens.tumblr.com/post/10153633521/how-to-participate-in-an-open-online-course . 2011

[8] http://open.163.com/

[9] http://www.icourses.cn/home/

[10] http://www.xuetangx.com/ 